\title{
Analysing the Effects of Mobility Model on QoS in MANET
}

\author{
Amit Garg \\ Ph.D. Scholar, IFTM Univ. \\ Moradabad \\ U.P. India
}

\author{
Ashish Kumar, $\mathrm{PhD}$ \\ Professor and Head, \\ I.T.S Engineering College, \\ Greater Noida, India
}

\author{
Amit Kr. Chaturvedi, PhD \\ Govt. Engineering College \\ Ajmer \\ Rajasthan, India
}

\begin{abstract}
As we know that in a Mobile Adhoc Network [MANET], mobility is the very important factor. Mobility gives the flexibility to the node to move in the coverage area. Here, in MANET multiple mobility models are proposed and every model provides different mobility and Quality of Service (QoS) support to the nodes. In this paper, we will present a study on these mobility models and its effects on QoS support in MANET and important findings.
\end{abstract}

\section{General Terms}

MANET, AODV, Routing Protocols, Mobility models, Random walk, random waypoint, QoS.

\section{Keywords}

MANET, AODV, Routing Protocols, Mobility models, Random walk, random waypoint, QoS

\section{INTRODUCTION}

Whenever a mobile adhoc network is designed, setting network environment parameters like, number of nodes, size of rectangular area, simulation time, Agent type, Application type, packet size, packet transfer rate, mobility model, maximum speed, pause time, and protocols etc, is the main requirement. Mobility Model allows the nodes or objects, how they behave in the system, their movement and the way they move. QoS is the ability to provide different priority to different applications, users, or data flows, or to guarantee a certain level of performance to a data flow. Quality of service guarantees are important if the network capacity is insufficient, especially for real-time streaming multimedia applications, since these often requires fixed bit rate and are delay sensitive and in network where the capacity is a limited resource

There are two types of MANET mobility models: singleentity and group. In single-entity models, each mobile node moves independently of all the other nodes within the network area i.e. the movement is independent to communication links between nodes. In group mobility models, nodes are assumed to be organized in groups and the mobility of a node is often reflective of the movement pattern of the entire group.

\section{VARIOUS MOBILITY MODELS USED IN AD-HOC NETWORKS}

To impersonate the movement of real mobile nodes that change the speed and direction with time, mobility model is the main source. The characteristics of the mobile nodes can be represented in adhoc network using mobility model. After defining the nodes characteristics or scenario, a network protocol may be examined for its usefulness in that network. There are two types of approaches used to model the mobility pattern : (1) traces, and (2) syntactic. If a mobility pattern is to be set by observing the real-life systems, traces are better option. In trace-based models, everything is deterministic. In absence of traces, the syntactic models that have been proposed to represent the movements of mobile nodes realistically in ad hoc networks are presented.

The syntactic mobility models can also be classified based on the description of the mobility patterns in ad hoc networks: individual mobile movements or single entity model and group mobile movements or group mobility model. In the case of individual mobile movements, mobility models attempt to the anticipate mobile's traversing patterns from one place to another at a given point of time under various network scenarios. In the case of group mobile movements, mobility models try to characterize the group's traversing patterns with individualism averaged.

Unlike trace-based mobility models, syntactic mobility models considered here have randomness, and further classifications can be made based on randomness: constrained topology-based models and statistical models. In constrained topology-based mobility models, mobile nodes have only partial randomness where the movement of nodes is restricted by obstacles, pathways, speed limits, and others. If the nodes are allowed to move anywhere in the area and the speed and direction are allowed to choose, it is termed as total randomness. The model that is based on total randomness is defined as statistical mobility model.

Based on specific mobility characteristics, the classification of mobility models is also made primarily into four categories: random models, models with temporal dependency, models with spatial dependency, and models with geographical restrictions. In random models, like statistical models, nodes move randomly and can be classified further based on the statistical properties of randomness, and random waypoint, random direction, and random walk mobility model fall into this category. The movement patterns of the mobility models with temporal dependency are likely to be influenced by their movement histories, and Gauss-Markov and smooth random mobility model are the examples of this mobility model category. In some mobility scenarios, the mobile nodes tend to travel in a correlated manner. These mobility models are termed as mobility models with spatial dependency, and mobility models like reference point group mobility model and other spatially correlated mobility models belong to this category. Another class is the mobility model with geographic restriction, same as the constrained topology-based model, 
where the movements of the mobile nodes are constrained by streets, freeways, and/or obstacles, and pathway and obstacle mobility model are two examples of this mobility model. Mobility models can also be categorized by using other criteria such as mobility patterns and histories: random mobility, directional mobility, and habitual mobility.

In mobility model activity of user's movement can be described using analytical and simulation models. Analytical models may provide performance parameters and Simulation models can derive valuable solutions for more complex cases. Typical mobility model includes:

1. Brownian Model

2. Random Waypoint Model

3. Random Walk Model

4. Random Direction Model

5. Random Gauss-Markov Model

6. Markovian Model

7. Incremental Model,

8. Mobility Vector Model

9. Reference Point Group Model (RPGM)

10. Pursue Model

11. Nomadic Community Model

12. Column Model

13. Fluid Flow Model

14. Exponential Correlated Random Model

15. Map Based Model

16. Manhattan Mobility Model

17. Mission Critical Mobility Model

18. Obstacle Mobility Model

19. Smooth Random Mobility Model

20. Post Disaster Mobility Model

Mobility models are chosen for simulation based on their different classes of motion as random based and group based movements.

1. Random based Mobility Models: In random based mobility models, the mobile nodes move randomly and freely without restrictions. The destination, speed and direction are all chosen randomly and independently of other nodes. The different types are discussed below:

A. Random Walk Model: It is the simplest and widely used model in MANET. It works on the concept of first differences on the random walk. First difference is the difference from one observation to the next. As we use a sequence of stumbling, unpredictable steps during the process of walking towards the goal. While walking there are the differences between each step and it has no reason. In a random walk model, the series itself is not random. However, its differences, the changes from one period to the next, are random. This difference may be shown by the equation,

$$
\mathrm{e}_{\mathrm{t}}=\mathrm{X}_{\mathrm{t}}-\mathrm{X}_{\mathrm{t}-1}
$$

Where, $e_{t}$ is the value of the error term in time period $t, X_{t}$ is the value in time period $t$, and $X_{t-1}$ is the value in time period $(\mathrm{t}-1)$.

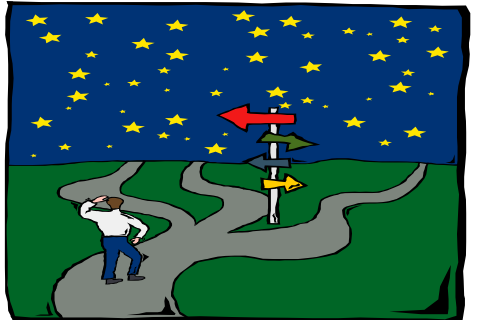

Figure 1: Random Walk

B. Random Waypoint Model : Random Waypoint (RWP) model is a commonly used synthetic model for mobility, e.g., in Ad Hoc networks. It is an elementary model which describes the movement pattern of independent nodes by simple terms.

Briefly, in the RWP model:

- Each node moves along a zigzag line from one waypoint $\mathrm{P}_{\mathrm{i}}$ to the next $\mathrm{P}_{\mathrm{i}+1}$.

- The waypoints are uniformly distributed over the given convex area, e.g. unit disk.

- At the start of each leg a random velocity is drawn from the velocity distribution. (in the basic case the velocity is constant 1 )

Optionally, the nodes may have so-called "thinking times" when they reach each waypoint before continuing on the next leg, where durations are independent and identically distributed random variables.

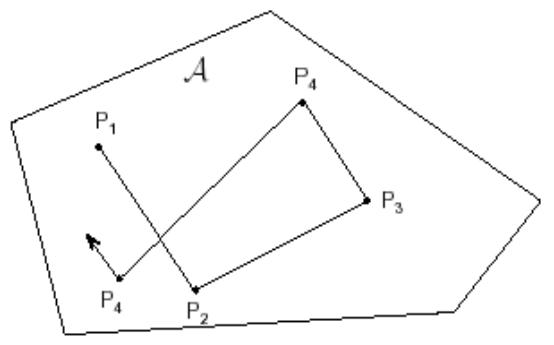

Figure 2: Movement of a node in Random Waypoint

\section{Common Problems with Random Waypoint Model}

- Zig-zag trajectories: RWP model is elementary and it is easy to argue about the paths being unnatural. Then again, any practical protocol or mechanism should be robust and give a reasonable performance with a wide range of moving patterns, including movement similar to RWP model.

- Velocity distribution: The most common problem with simulation studies using random waypoint model is a poor choice of velocity distribution [5], e.g., uniform distribution $\mathrm{U}(0, \mathrm{Vmax})$. Such velocity distributions (which seem to be common with NS-2 simulations!) lead to a situation where at the stationary state each node stops moving. In order to avoid this the velocity distribution should be such that

$$
1 / \mathbf{E}[\mathbf{1} / \mathrm{V}]>0
$$

Note that the mean time a node spends on a single leg is proportional to $1 / \mathrm{E}[1 / \mathrm{V}]$.

B(i). Random Waypoint on the Border (RWPB) : In the (standard) RWP model the waypoints are uniformly distributed over the given domain. Alternatively, the waypoints can be uniformly distributed on the border of the domain and this model is referred to as the "Random waypoint on the border" (RWPB) model. The spatial node density resulting from RWPB model is quite different from the RWP 
model, i.e. the probability mass shifts from the center of the area to the borders. In particular, if the border contains a straight line segment, then there is a positive probability that a random leg resides on the line segment (resulting in a 1dimension pdf on each line segment on the border).

B(ii). Markovian Waypoint Model (MWP) : The RWP model can be further extended, e.g., by allowing the next waypoint depend on the current waypoint. This leads to a socalled Markovian Waypoint Model (MWP) [11], where one can also define leg depending velocity distributions (velocity from P1 to P2 is no longer i.i.d. random variable but depends on $\mathrm{P} 1$ and $\mathrm{P} 2$ ) and random pause times during the transition between two waypoints.

C. Random Direction Model : The random direction mobility model is, besides the random waypoint model, probably the most widely used synthetic mobility model for mobile communications research. As well as the RWP model, this model considers individuals moving on straight walk segments with constant speed and optional pauses between walk segments. There are several flavours of the random direction model which slightly differ in the way they obtain the next walk segment. Hong and Rappaport [9] propose a model that is build on top of a cell structure and apply walkers that pass those cells on straight lines and choose new directions at cell borders. Gu'erin [10] extends this model in a way that direction changes can be performed anywhere in a walk area. Some approaches model the direction choice with absolute angles while others like the one proposed by Zanoozi [11] calculate with relative changes to the current direction.

2. Group Mobility Model: Group mobility model represents multiple mobile nodes whose actions are completely independent of each other. For example, a group of soldiers in a military scenario may be assigned the task of searching a particular plot of land in order to destroy land mines. In order to model such situations, a group mobility model is needed to simulate this kind of characteristic. Here each group has a logical centre (group leader) that determines the group's motion behavior. Initially each member of the group is uniformly distributed in the neighbourhood of the group leader. Subsequently, at each instant, every node has speed and direction that is derived by randomly deviating from that of the group leader. Each node deviates from its velocity (both speed and direction) randomly from that of the leader.

\section{LITERATURE SURVEY ON QOS IN MANET}

J. Abdullah discussed the performance of QOSRGA Routing Protocol for MANET with Random Waypoint Mobility Model. A scheme has been presented for multiple constrained QoS routing protocol for MANET based on Genetic Algorithm. In the proposed scheme of QoS routing, selection of a route was based on node bandwidth availability, short end to end delay and the longest node pair connectivity time indicated by node connectivity index (nci). The route selection algorithm was outlined and implemented. The variable length chromosomes represented the routes and genes represented the nodes. The algorithmic process was initialised by introducing a limited population, accumulated during the route discovery by the Node non-Disjoint Multiple Route Discovery (NDMRD) protocol. The fitness calculation was done using the weighted sum approached, combining the entire objective functions into a single objective. The scenario used the Random Waypoint Mobility model for ensuring that the nodes movement in a random fashion. The performance study was done to study the effect of maximum node velocity on the average packet delivery ratio and delay. The performances indicated that the protocol is feasible for a reasonable node velocity with random mobility model [1].

D.S. Devi and Dr. G. Padmavathi, discussed the Impact of Mobility for QoS Based Secure MANET. Secure multicast communication in Mobile Adhoc Networks (MANETs) is challenging due to its inherent characteristics of infrastructure-less architecture with lack of central authority, limited resources such as bandwidth, energy and power. Several group oriented applications over MANETs create new challenges to routing protocols in terms of QOS requirements. In many multicast interactions, due to its frequent node mobility, new member can join and current members can leave at a time. It is necessary to choose a routing protocol which establishes true connectivity between the mobile nodes. The pattern of movement of members is classified into different mobility models and each one has its own distinct features. It is a crucial part in the performance of MANET. Hence key management is the fundamental challenge in achieving secure communication using multicast key distribution for mobile adhoc networks. This paper describes the impact of mobility models for the performance of a new cluster-based multicast tree algorithm with Destination Sequenced Distance Vector (DSDV) routing protocol in terms of QOS requirements such as end to end delay, energy consumption and key delivery ratio. For simulation purposes, three mobility models are considered. Simulation results illustrate the performance of routing protocol with different mobility models and different mobility speed under varying network conditions [2].

Anton Cizmar, Jan Papaj, Lubomır Dobos, proposed security and QOS integration model for MANETS. The new model used to integrating security and Quality of Service $(\mathrm{QoS})$ as one parameter in mobile ad-hoc network (MANET) is introduced and studied in this article. Security and QoS represent a highly important field of research in MANET and they are still being considered separately with no mechanisms used to establish cooperation between them. This new model provides alternative to cooperation between QoS and security via cross layer design (CLD) and modified security service vector. Performance analysis of the new designed model is introduced too. It is also considered herein how processing of the new integrating model affects the performance of the MANET networks [3].

I. Vijaya, Amiya Kumar Rath, Bhagabat Puthal, Debahuti Mishra and S. Satapathy, presented Performance Analysis of QoS Parameters of MANET on Mobility and Energy based Model with Different MANET Routing Protocols. A network is group of devices that are connected to each other called as nodes. The nodes can be mobile or static. The performance of mobile Adhoc wireless networks (MANETs) helps to identify the type of applications that are supported by the network. Our objective is Performance analysis of QoS parameters of MANETs on Mobility \& Energy based Model with Routing Protocols. The various network scenarios of MANETS are simulated using NS2.35. Protocols used to analyze performance are AODV, DSDV and DSR. Network layer parameters (throughput, packet delivery ratio, normalized routing overhead and average end-to-end delay) are evaluated. Network scenarios are generated through variation in pause time and number of nodes. Area of simulation is formed in $600 * 600 \mathrm{~m} * \mathrm{~m}$ area. The mobiles devices in the network get 
connected only when there is a demand for it. The reactive gateway discovery algorithm is used in AODV and DSR. With the random movement of nodes in the simulated area (direction) and variation in mobility, the delay and packet drop increases but PDR and throughput decreases. There is a significant differential observed while measuring the performance. Our observation with respect to DSR was it reacted well for two parameters delivery ratio and routing overhead. Average delay was less in AODV and DSDV performed well providing loop free path. After the simulation study and all experimental evaluations we can conclude that the DSR protocol dominates all other protocols like AODV and DSDV. The Dynamic Source Routing protocol in mobility and energy based model for throughput, packet delivery ratio performs well than AODV and DSDV. The adverse result is with the increase of node speed, routing overhead increased for DSR. Positive aspect of DSR was that average energy consumption was quite low in contrast to AODV and DSDV [4].

Yee Leung, Guo Li, and Zong-Ben $\mathrm{Xu}$, proposed a Genetic Algorithm for the Multiple Destination Routing Problems. The multiple destination routing (MDR) problem can be formulated as finding a minimal cost tree which contains designated source and multiple destination nodes so that certain constraints in a given communication network are satisfied. This is a typical NP-hard problem, and therefore only heuristic algorithms are of practical value. As a first step, a new genetic algorithm is developed to solve the MDR problems without constraints. It is based on the transformation of the underlying network of an MDR problem into its distance complete form, a natural chromosome representation of a minimal spanning tree (an individual), and a completely new computation of the fitness of individual. Compared with the known genetic algorithms and heuristic algorithms for the same problem, the proposed algorithm has several advantages. First, it guarantees convergence to an optimal solution with probability one. Second, not only are the resultant solutions all feasible, the solution quality is also much higher than that obtained by the other methods (indeed, in almost every case in our simulations, the algorithm can find the optimal solution of the problem). Third, the algorithm is of low computational complexity, and this can be decreased dramatically as the number of destination nodes in the problem increases. The simulation studies for the sparse and dense networks all demonstrate that the proposed algorithm is highly robust and very efficient in the sense of yielding high-quality solutions [5].

Said EL KAFHALI, Abdelkrim HAQIQ, presented the effect of Mobility and Traffic Models on the Energy Consumption in MANET Routing Protocols. A Mobile Ad hoc Network (MANET) is a group of mobile nodes that can be set up randomly and formed without the need of any existing network infrastructure or centralized administration. In this network the mobile devices are dependent on battery power, it is important to minimize their energy consumption. Also storage capacity and power are severely limited. In situations such as emergency rescue, military actions, and scientific field missions, energy conservation plays an even more important role which is critical to the success of the tasks performed by the network. Therefore, energy conservation should be considered carefully when designing or evaluating ad hoc routing protocols. In this paper we concentrated on the energy consumption issues of existing routing protocols in MANET under various mobility models and whose connections communicate in a particular traffic model (CBR, Exponential, and Pareto). This paper describes a performance comparison of the AODV, DSR and DSDV routing protocols in term of energy consumed due to packet type (routing/MAC) during transmission and reception of control packets. The mobility models used in this work are Random Waypoint, Manhattan Grid and Reference Point Group. Simulations have been carried out using NS-2 and its associated tools for animation and analysis of results [6].

D. Johnson, D. Maltz, D. Maltz, proposed the Dynamic Source Routing Protocol (DSR) for Mobile Ad Hoc Networks for IPv4. The Dynamic Source Routing protocol (DSR) is a simple and efficient routing protocol designed specifically for use in multi-hop wireless ad hoc networks of mobile nodes. DSR allows the network to be completely self-organizing and self-configuring, without the need for any existing network infrastructure or administration. The protocol is composed of the two main mechanisms of "Route Discovery" and "Route Maintenance", which work together to allow nodes to discover and maintain routes to arbitrary destinations in the ad hoc network. All aspects of the protocol operate entirely on demand, allowing the routing packet overhead of DSR to scale automatically to only what is needed to react to changes in the routes currently in use. The protocol allows multiple routes to any destination and allows each sender to select and control the routes used in routing its packets, for example, for use in load balancing or for increased robustness. Other advantages of the DSR protocol include easily guaranteed loop free routing, operation in networks containing unidirectional links, use of only "soft state" in routing, and very rapid recovery when routes in the network change. The DSR protocol is designed mainly for mobile ad hoc networks of up to about two hundred nodes and is designed to work well even with very high rates of mobility. This document specifies the operation of the DSR protocol for routing unicast IPv4 packets [7].

Mohamed Amnai, Youssef Fakhri, Jaafar Abouchabaka, discussed the Impact of Mobility on Delay-Throughput Performance in Multi-Service Mobile Ad-Hoc Networks[8]. S.R. Biradar, S.K. Sarkar, Rajanna KM, Puttamadappu C., presented Analysis QoS Parameters for MANETs Routing Protocols. A Mobile Ad-Hoc Network (MANET) is a decentralized network of autonomous mobile nodes able to communicate with each other over wireless links. We selected three routing protocols DSDV, DSR and AODV for measuring QoS parameters. We have used the network simulator ns-2 for simulating routing protocols using group mobility model, and present the results of simulations of networks of 40 wireless mobile nodes [9].

Keerthi D.S, Chaithanyaranga H V, Shreedevi P, presented the Study and Performance Evaluation of Mobility Models in MANET and WSN. The impact of various mobility models like flag mobility and random waypoint mobility models on QoS issues in MANET and WSN are studied. The performance of the MANET and WSN are evaluated for the mobility models using QualNet 6.1 simulator [10]. Fahim Maan, N. Mazhar, presented the MANET Routing Protocols vs Mobility Models: A Performance Evaluation. The fundamental characteristic which differentiates MANETs from other wireless or wired networks is mobility. They state that our simulative study on MANET routing protocols and mobility models aims to determine the performance of current MANET routing protocols with respect to various mobility models implemented in ns-2. We compare a number of reactive and proactive routing protocols including AODV, 
DSR, DSDV, OLSR and DYMO. The results of our extensive network simulations are tabulated along with a comprehensive analysis. The effort allows a fair comparison of the capabilities and limitations of different types of mobility patterns and their suitability for contemporary MANET routing protocols [11].

A.K.Chaturvedi, J.k.Khemani, presented Analysis of Mobility Models in Mobile Ad-hoc Networks. Mobility models in adhoc networks uses protocols to discover and setup routes between nodes [12]. Hannan XIAO, Winston K.G. Seah, Anthony LO, and Kee Chaing CHUA, proposed a flexible quality of Service Model for Mobile Ad-Hoc Networks. QoS supports in MANET is challenging task. Most of the proposals in the literature only address certain aspects of the QoS support, e.g., QoS routing, QoS medium access control (MAC) and resource reservation. However, none of them proposes a QoS model for MANETs. Meanwhile, two QoS models have been proposed for the Internet, viz., the Integrated Services (IntServ) Model and the Differentiated Service (DiffServ) model, but these models are aimed for wired networks. In this paper, we propose a flexible QoS model for MANETs (FQMM), which considers the characteristics of MANETs and combines the high quality QoS of IntServ and service differentiation of DiffServ. [13].

\section{IMPORTANT FINDINGS}

After going through the related literature survey, we find some useful information regarding the adhoc networks, security service cases, QoS requirements like key management, multicast communications, movement patterns, and performance evolution. These useful information are listed below:

The combination of an adhoc environment with multicast services induces new challenges towards the security infrastructure. In order to secure multicast communication, security services such as authentication, data integrity, access control and group confidentiality are required. These security services can be facilitated if group members share a common secret, which in turn makes key management a fundamental challenge in designing secure multicast and reliable group communication systems. Group confidentiality requires that only valid users could decrypt the multicast data. This can be done using key distribution rules: (1) Non-group confidentiality, (2) Forward secrecy, (3) Backward secrecy, and (4) Collusion freedom.

Most of these security services rely generally on encryption using Traffic Encryption Keys (TEKs). The Key management includes creating, distributing and updating the keys then it constitutes a basic block for secure multicast communication applications. The process of updating the keys and distributing them to the group members is called rekeying operation. A critical problem with any rekey technique is scalability. The rekey process should be done after each membership change, and if the membership changes are frequent, key management will require a large number of key exchanges per unit time in order to maintain both forward and backward secrecies. The number of TEK update messages in the case of frequent join and leave operations induces several QOS characteristics.

Thus a secure multicast key distribution in mobile ad hoc environment should focus on both security and Qos characteristics. A cluster based multicast tree (CBMT) algorithm for secure multicast key distribution in mobile adhoc networks. The frequent mobility of members and limited communication resources make routing in MANET very difficult. Mobility causes frequent topology changes and may break existing paths. A routing protocol should quickly adapt to the topology changes and efficiently search for new paths. To overcome these above limitations, Destination Sequenced Distance Vector routing protocol is used. It allows fast reaction to topology changes and is specially designed for MANET.

The pattern of movement of members can be classified into different mobility models and each is characterized by their own distinct features. The traditional mobility models includes (i) Random Waypoint Model (ii) Random Walk Model and (iii) Group Mobility Model which are simple to implement and analyze. These are randomized model in which each member chooses their velocity and direction independently without any restrictions. Hence these models do not capture correlation between the member movements. Recent work on mobility models attempts to identify common mobility movement. The CBMT (Cluster Based Multicast Tree) approach is an efficient dynamic clustering scheme using DSDV routing protocol, which makes easy to elect the local controllers of the clusters and updates periodically as the node joins and leaves the cluster.

The notion of Quality of Service (QoS) is a guarantee provided by the network to satisfy a set of predetermined service performance constraints for the user in terms of the end-to-end delay statistics, available bandwidth, probability of packet loss, etc. [1]. There are many applications and services that require specific QoS guarantees. In literature, the research of QoS support in MANETs includes:

- QoS models - specifying an architecture in which some kinds of services could be provided.

- QoS routing - a part of the network layer, searches for a path with enough resources but does not reserve resources.

- QoS adaptation - hides all environment-related features from awareness of the multimedia application above and provides an interface for applications to interact with QoS control.

- QoS signalling acts - a control centre in QoS support. Functionality of QoS signalling is determined by the QoS model.

- QoS MAC protocols - essential components of QoS for MANETs. MAC protocols solve the problems of medium contention, support reliable communication, and provide resource reservation.

The performance evaluation of routing protocols under various mobility models provides a basis for selection of MANET protocols to meet specific network scenarios. Table 1 presents the matrix of guidelines for selected the routing protocols with a particular mobility model. The results presented in Table 1 indicate that AODV has a higher performance under RWP model for small networks. Consequently, we may choose AODV for small networks with RWP as the mobility model. Similarly, large AODV networks with RPGM as mobility model perform better. So we now have a scale to select a particular protocol according to our selection of the network performance parameter. Secondly, we select a routing protocol if it outperforms in any two mobility models for a single performance parameter. These routing protocols are our second best candidates. Thirdly, we look for protocols that give the best performance for different parameters under different mobility models. 
These protocols are the third best candidates for selection as a routing agent in a MANET. Table 1 shows the groups of protocols for the three selection criteria in boxes of varying shades of grey.

\begin{tabular}{|c|c|c|c|c|c|c|c|c|c|c|c|c|}
\hline \multirow{2}{*}{ 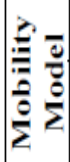 } & \multicolumn{3}{|c|}{25} & \multicolumn{3}{|c|}{50} & \multicolumn{3}{|c|}{75} & \multicolumn{3}{|c|}{100} \\
\hline & $\overline{\bar{z}}$ & 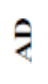 & $\frac{\underline{O}}{\hat{Q}}$ & $\overline{\bar{z}}$ & $\hat{z}$ & $\frac{\underline{x}}{\hat{a}}$ & $\overline{\bar{z}}$ & e & $\frac{x}{0}$ & $\overline{\bar{z}}$ & 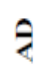 & $\frac{\mathrm{x}}{\hat{Q}}$ \\
\hline$\frac{0}{\underline{z}}$ & $\frac{\frac{d}{n}}{\frac{1}{0}}$ & \%) & 常 & $\begin{array}{l}\frac{x}{n} \\
\frac{1}{0}\end{array}$ & $\begin{array}{l}0 \\
\sum_{\lambda} \\
0\end{array}$ & $\stackrel{\overrightarrow{0}}{\overrightarrow{0}}$ & $\begin{array}{l}\frac{x}{n} \\
\frac{0}{0}\end{array}$ & $\sum_{\substack{0 \\
0}}^{0}$ & $\overrightarrow{\mathrm{o}}$ & $\begin{array}{l}\frac{x}{w} \\
\frac{1}{0}\end{array}$ & )े & 总 \\
\hline $\begin{array}{l}\sum \\
\vdots \\
\underline{\Delta}\end{array}$ & 尚 & $\frac{\frac{v}{b}}{0}$ & $\frac{\alpha}{n}$ & ) & ) & $\frac{\alpha}{w}$ & $\stackrel{3}{0}$ & $\sum_{0}^{0}$ & $\frac{\frac{\alpha}{a}}{\frac{1}{0}}$ & ô & \% & 定 \\
\hline$\sum_{U}^{\Sigma}$ & 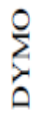 & $\frac{\frac{x}{w}}{0}$ & $\frac{x}{n}$ & ) & $\sum_{0}^{0}$ & 炃 & $\begin{array}{l}> \\
0 \\
0\end{array}$ & 8 & 2) & $\frac{3}{0}$ & $\sum_{0}^{0}$ & $\frac{\frac{\alpha}{n}}{0}$ \\
\hline
\end{tabular}

Table 1: Matrix For Selection Of Routing Protocols In Terms Of Mobility Models And Performance Parameters

The terms used in the table are described below:

\begin{tabular}{|ll|}
\hline$\bullet$ & CMM [Column Mobility Model] \\
$\bullet$ & Reference Point Group Mobility (RPGM) \\
\hline & Random Waypoint Model (RWP) \\
\hline & Optimized Link State Routing Algorithm (OLSR) \\
- & Dynamic MANET on demand (DYMO) \\
\hline & Adhoc on Demand Distance Vector (AODV) \\
\hline & Packet Delivery Ratio (PDR) \\
- Average Delay(AD) \\
- Pormalized Routing Load (NRL) \\
\hline
\end{tabular}

\section{CONCLUSION}

It is evident from the results that AOMDV (Ad Hoc On Demand Multipath Distance Vector) protocol perform better in term of PDR in Probabilistic Random walk model in low node mobility, and for higher node mobility except random direction model in other models PDR decreases. The DSR protocol dominates all other protocols like AODV and DSDV. The Dynamic Source Routing protocol in mobility and energy based model for throughput, packet delivery ratio performs well than AODV and DSDV. Positive aspect of DSR was that average energy consumption was quite low in contrast to AODV and DSDV.

The impact of different mobility models on the performance of CBMT (Cluster Based Multicast Tree) approach with DSDV routing protocol varies widely across different number of nodes and node mobility speed in terms of QOS performance metrics as average end to end delay, energy consumption key delivery ratio and routing overhead for secure MANETs. It is observed that the movement of nodes is characterized based on mobility speed. It is observed that the Random waypoint produces better results in suitable conditions than the other two mobility models in such adhoc environment.
We have observed the behavior of MANET routing protocols under three mobility models (RWP, RPGM and CMM and found that an increase in network size and number of nodes has similar impact on all protocols under various mobility patterns. However, the degree of degradation varies for different combinations of protocols and mobility models.

\section{ACKNOWLEDGMENTS}

We thanks to all the contributors directly or indirectly towards the development of this paper.

\section{REFERENCES}

[1] J.Abdullah," Performance of QOSRGA Routing Protocol for MANET with Random Waypoint Mobility Model", International Journal of Advanced Science and Technology, Vol. 40, March, 2012, pp. 19-34.

[2] D.S.Devi and Dr. G. Padmavathi, "Impact of Mobility for QoS Based Secure MANET", International journal on applications of graph theory in wireless ad hoc networks and sensor networks, (GRAPH-HOC) Vol.2, No.3, September 2010, pp 46-57.

[3] Anton Cizmar, Jan Papaj, Lubomir Dobos, "SECURITY AND QOS INTEGRATION MODEL FOR MANETS", Computing and Informatics, Vol. 31, 2012, pp. 10251044.

[4] I. Vijaya, Amiya Kumar Rath, Bhagabat Puthal, Debahuti Mishra and S. Satapathy, "Performance Analysis of QoS Parameters of MANET on Mobility and Energy based Model with Different MANET Routing Protocols", Indian Journal of Science and Technology, Vol 9(37), DOI: 10.17485/ijst/2016/v9i37/100468, October 2016, ISSN (Print) : 0974-6846, ISSN (Online) : 0974-5645

[5] Yee Leung, Guo Li, and Zong-Ben $\mathrm{Xu}$, "A Genetic Algorithm for the Multiple Destination Routing Problems", IEEE TRANSACTIONS ON EVOLUTIONARY COMPUTATION, VOL. 2, NO. 4 NOVEMBER 1998.

[6] Said EL KAFHALI, Abdelkrim HAQIQ, "Effect of Mobility and Traffic Models on the Energy Consumption in MANET Routing Protocols", International Journal of Soft Computing and Engineering (IJSCE), ISSN: 22312307, Volume-3, Issue-1, March 2013

[7] D. Johnson, D. Maltz, D. Maltz, "The Dynamic Source Routing Protocol (DSR) for Mobile Ad Hoc Networks for IPv4", RFC 4728, February 2007

[8] Mohamed Amnai, Youssef Fakhri, Jaafar Abouchabaka, "Impact of Mobility on Delay-Throughput Performance in Multi-Service Mobile Ad-Hoc Networks", Int'1 J. of Communications, Network and System Sciences, 2011, 4, 395-402 , doi:10.4236/ijcns.2011.46047 Published Online June 2011.

[9] S.R. Biradar, S.K. Sarkar, Rajanna KM, Puttamadappu C., "Analysis QoS Parameters for MANETs Routing Protocols", International Journal on Computer Science and Engineering, Vol. 02, No. 03, 2010, pp. 593-599.

[10] Keerthi D.S, Chaithanyaranga H V, Shreedevi P," Study and Performance Evaluation of Mobility Models in MANET and WSN", 2016 International Conference on Electrical, Electronics, Communication, Computer and 
Optimization Techniques (ICEECCOT), ISBN : 978-15090-4697-3/16/\$31.00 @2016 IEEE, pp. 297-301.

[11] Fahim Maan, N. Mazhar, "MANET Routing Protocols vs Mobility Models: A Performance Evaluation", ICUFN 2011, ISBN : 978-1-4577-1177-0/11/\$26.00 (02011 IEEE, pp. 179-184.

[12] A.K.Chaturvedi, J.k.Khemani, "Analysis of Mobility Models in Mobile Ad-hoc Networks", presented in
NWNC-2014, proceeding published in IJCA ISSN: 0975-8887, pp.5-9.

[13] Hannan XIAO, Winston K.G. Seah, Anthony LO, and Kee Chaing CHUA, "A flexible quality of Service Model for Mobile Ad-Hoc Networks", VTC 2000, ISBN : 07803-5718-3,

pp. 445-449. 